\title{
Analysis of the Dynamic Characteristics of Seat Headrest During Impact
}

\author{
JIANG Tao ${ }^{1, a}$, ZHANG GuiLin ${ }^{1, b^{*}}$, Li min ${ }^{2, c}$, Li huiming ${ }^{1, d}$ \\ ${ }^{1}$ Changchun University of Science and Technology, 7186 Weixing Road, Changchun, Jilin, 130022 \\ china \\ ${ }^{2}$ Changchun MOIRE Electronics Co.,LTD, 358 Zhenyu Street, Changchun, Jilin, 130012 china \\ ajt12345@126.com, bguilin512400@126.com, chlgIm@126.com, ${ }^{\mathrm{d}} \mathrm{l}$ hming52125@sina.com
}

Keywords: Seat headrest; Dynamic characteristics; Three component-force detection

Abstract. A energy-absorber device with gravity pendulum of car seat headrest is introduced in this paper, and has a analysis at the dynamic characteristics of headrest by use the method of three component-force detection. A force measuring system from the three component-force detection is brought up to reseach the model error in impact model, and obtained the point, which the model has a minimum error. Finally, the impact model was simulated with MATLAB by computer, as a results show that the simulattion curves are similar to the real response curves very much.

\section{Introduction}

Due to the important of safety and convenience, car seats become an object of study with the advent of the car in automakers. In collision accident, whiplash is one of the major damage for occupant. Thanks to the emergence of the car seat headrest, the economic and casualties caused by traffic accident has greatly reduced. The principle of reduce damage could describe as by impose restrictions on occupant head, the relative displacement between the head and body is limited, it ensures the reduction of the whiplash. Therefore, as a key part of automobile seat, the technical requirements for seat headrest is the most severe in required and a lot of experiments is necessary, before headrest leaving the factory ${ }^{[1]}$. Because of the dynamic performance of the seat headrest during impact, is affect on seat safety directly, energy absorbed properties became one of the most important bases for evaluating the quality of seat headrest. In this paper, a second order control model is established to analysis the dynamic performance of the headrest, lay the foundation for follow-up study by confirm the appropriate parameters in simulation.

\section{The Basic Structure and Principle}

The device of impact testing. The device of seat headrest impact testing can be classified in two types according to impact energy access: on the basis of ejection, a power soure with hydraulic or pneumatic was carried out on the headrest ${ }^{[2,3]}$; on the basis of potential energy transduction principle, provide the impact energy by gravity pendulum ${ }^{[4,5]}$. However, each one of these prime mover are aim to get an instantaneous impact at seat headrest, in this paper, pendulum impact testing machine is the object of research platform.

Among mang detection techniques of dynamic characteristics of the seat headrest, because of the structure of the headrest, the different position, and the uncertainty of the impact point, the impact force become difficulties in testing ${ }^{[6]}$. The method, which called three component-force detection, is commonly used in single point impact detect, is introduced as the basic analysis theory of the headrest ${ }^{[7]}$.

As it is shown in figure 1, typically, an energy absorbing machine of automobile seats, which mainly consists of angle sensor, pendulum pole, head model, motor, seats, support and the foundation. In actual work, by the driving of the motor swing rod turning an angle, and make the pendulum to rise a certain height, gravitational potential energy will transforms into impact energy, when motor released, to complete the absorption test of the seat ${ }^{[8]}$. 


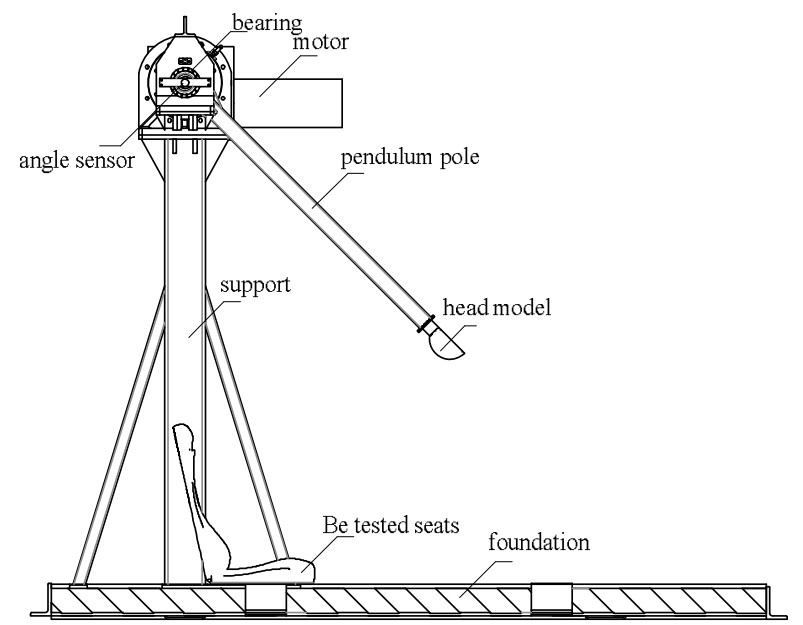

Fig. 1 Structure of energy absorbing machine

Model of impact testing. The method of three component-force detection, in order to reduce the error caused by impact of uncertainty, there are three pressure sensor evenly place in a plane with $120^{\circ}$. Take the interface between the headrest and pendulum as a zero thickness and weight circular plate, and take the headrest as a mass spring damping system, establishes a mathematical model as it is shown in figure 2.

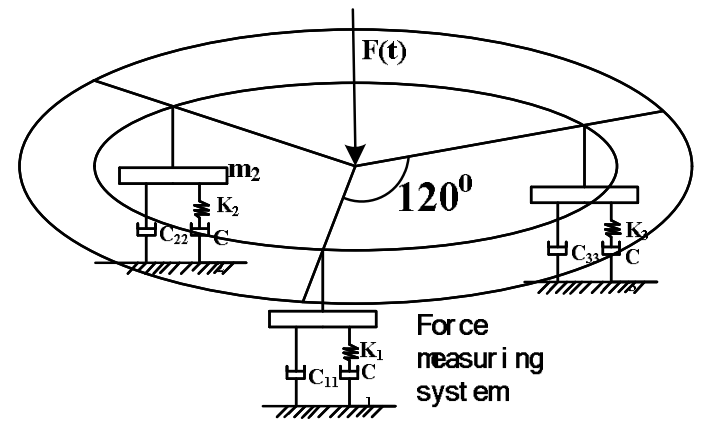

Fig. 2 The model of three component-force detection

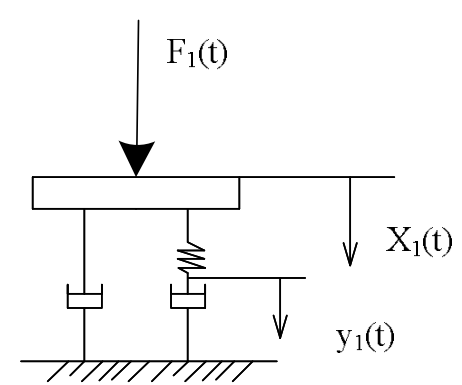

Fig. 3 Force measuring system

In Fig. $2, F(t)$ is the impact in seat headrest, $\mathrm{k}_{1}, \mathrm{k}_{2}, \mathrm{k}_{3}$ is three pring stiffness and $\mathrm{C}_{1}, \mathrm{C}_{11}, \mathrm{C}_{2}, \mathrm{C}_{22}$ 、 $\mathrm{C}_{3} 、 \mathrm{C}_{33}$ is the six damping coefficient in each fore measuring system. For comparison, as shown Fig.3, a monomer force measuring system was selected as a study subjects, $F_{1}(t)$ is one of the force, impacting in headrest, and $\mathrm{X}_{1}(\mathrm{t}), \mathrm{y}_{1}(\mathrm{t})$ is the displacement of seat headrest and spring. According to the theory of dynamics, we can reach $F(\mathrm{t})=F(\mathrm{t})+F_{2}(\mathrm{t})+F_{3}(\mathrm{t})$.

For the force measuring system, has an equation of state:

$$
\left\{\begin{array}{l}
k_{1}\left[x_{1}(t)-y_{1}(t)\right]=c y_{1}^{\prime}(t) \\
F(\mathrm{t})=m_{1} x_{1}^{\prime \prime}(t)+c x_{1}^{\prime}(t)+k_{1}\left[x_{1}(t)-y_{1}(t)\right]
\end{array}\right.
$$

With simplified and Laplace transform, the relationship between input impact and output displacement can be drawn from Eq. 1:

$$
G_{1}(\mathrm{~s})=\frac{F_{1}(\mathrm{t})}{X(\mathrm{t})}=\frac{\mathrm{c}_{1} \mathrm{~s}+\mathrm{k}_{1}}{m_{1} c_{1} s^{3}+\left(k_{1} m_{1}+c_{1}^{2}\right) s^{2}+2 c_{1} k_{1} s}
$$

Without losing generality, Eq. 2 could be describe as

$$
G_{1}(\mathrm{~s})=\frac{\mathrm{s}+z_{1}}{s\left(s^{2}+2 \xi w_{n 1}+w_{n 1}{ }^{2}\right)}
$$


Where, the natural frequencies was derived by $\mathrm{w}_{\mathrm{n} 2}=\sqrt{\frac{k_{2}}{m_{2}}}$, and damping ratio was derived by $\xi_{1}=\frac{c_{1}}{\sqrt{m_{1} k_{1}}}+\frac{\sqrt{m_{1} k_{1}}}{c_{1}}$, and there has a position of zero in $z=\frac{k_{1}}{c_{1}}$.

The same force measuring system 2 and 3 may be easily adapted to obtain:

$$
\left\{\begin{array}{l}
G_{2}(\mathrm{~s})=\frac{\mathrm{s}+z_{2}}{s\left(s^{2}+2 \xi w_{n 2}+w_{n 2}{ }^{2}\right)} \\
G_{3}(\mathrm{~s})=\frac{\mathrm{s}+z_{2}}{s\left(s^{2}+2 \xi w_{n 3}+w_{n 3}{ }^{2}\right)}
\end{array}\right.
$$

Where, as the same to the foce measuring system1, there natural frequencies and damping ratio were derived by:

$$
\left\{\begin{array}{c}
\mathrm{w}_{\mathrm{n} 2}=\sqrt{\frac{k_{2}}{m_{2}}} \quad \mathrm{w}_{\mathrm{n} 3}=\sqrt{\frac{k_{3}}{m_{3}}} \\
\xi_{2}=\frac{c_{2}}{\sqrt{m_{2} k_{2}}}+\frac{\sqrt{m_{2} k_{2}}}{c_{2}} \quad 3=\frac{c_{3}}{\sqrt{m_{3} k_{3}}}+\frac{\sqrt{m_{3} k_{3}}}{c_{3}}
\end{array}\right.
$$

And, their position of zero is $z_{2}=\frac{k_{2}}{c_{2}}$ and $z_{3}=\frac{k_{3}}{c_{3}}$.

\section{The model analysis}

The error analysis of the model. From the point of detection, the impacting in headrest is a transient signal, and the exciting source is a pulse signal. Simplified, assuming input function to be impules, we can reach the response function of the force measuring systems as: $X_{\text {on }}(\mathrm{s})=G_{\mathrm{n}}(\mathrm{s}) X(\mathrm{~s}), n=1,2,3$, and there has $X_{\mathrm{i}}=L[\delta(\mathrm{t})]=1, X_{\text {on }}(s)=G_{n}(s)$, therefore, colud obtain a further decomposed as shown in Eq.6 for response function:

$$
X_{\mathrm{oi}}=\frac{A_{i}}{\mathrm{~s}}+\frac{B_{i} s+C_{i}}{s^{2}+2 \xi_{i} w_{n i}+w_{n i}{ }^{2}}, i=1,2,3
$$

Where, $A_{i}=1 ; B_{i}=-1 ; C_{i}=2 \xi w_{n i}-1$ are constant determined by partial fraction. Further, as it described in Eq. 7, the unit pulse response of force measuring system is derived by using the inverse Laplace transform from Eq. 6:

$$
x_{n}(t)=A_{i}+\sqrt{1+\left(\frac{3 \xi_{i} w_{n i}-1}{w_{n i}}\right)^{2}} \exp \left(-\xi_{i} w_{n i} t\right) \sin \left[w_{n i} t+\arctan \left(\frac{w_{n i}}{1-\xi_{i} w_{n i}}\right)\right]
$$

As it known, the three force measuring system in model of seat headrest impact test, has the same model features. So that, take one of their as the measurement reference, while, the dispalacement errors of the detection system, caused by the uncertainty of the impact point and the inhomogeneity of dynamic characteristics between pressure sensor and the plate, have been obtained:

$$
e=\frac{x_{i}(t)-x_{1}(t)}{x_{1}(t)}, i=2,3
$$


To obtain the peak error of the model, take the derivative of the Eq. 7, reach the peak value as it shown in Eq. 9:

$$
x_{n}\left(t_{\max }\right)=A_{i}+\sqrt{1+\left(\frac{3 \xi_{i} w_{n i}-1}{w_{n i}}\right)^{2}} \exp \left\{-\xi_{i} \times\left[\arctan \left(\frac{1}{\xi_{i}}\right)-\arctan \left(\frac{w_{n i}}{1-\xi_{i} w_{n i}}\right)\right]\right\} \sin \left(\arctan \frac{1}{\xi_{i}}\right)
$$

Take other two force measuring system into the displacement error consider, the displacement error between the force measuring system 1 and forc measuring system 2 can been obtained by substituting response function of force measuring system 2 into the Eq. 8:

$$
e=\frac{\sqrt{1+\xi_{1}{ }^{2}} \exp \left[\xi_{1} \arctan \left(\frac{1}{\xi_{1}}\right)+\arctan \left(\frac{w_{1}}{\xi_{1} w_{1}-1}\right)\right] * \sqrt{\left(\frac{3 w_{2} \xi_{2}-1}{w_{2}}\right)^{2}+1}}{\sqrt{1+\xi_{2}{ }^{2}} \exp \left[\xi_{2} \arctan \left(\frac{1}{\xi_{2}}\right)+\arctan \left(\frac{w_{2}}{\xi_{2} w_{2}-1}\right)\right] * \sqrt{\left(\frac{3 w_{1} \xi_{1}-1}{w_{1}}\right)^{2}+1}}-1
$$

According the Eq. 10, it can be deduced that, if the force measuring systems are statistical consistency, the error $e$ can eliminated, but in the practical engineering, the errors are always exists, and we can determine the parameters through choose the right error values.

The simulation of the model. For conform with the actual situation, define $\mathrm{a}=\xi_{n} / w_{n}, n=1,2,3$ as a proportional between the natural frequencies and damping ratio. Assuming that, a has a respective value at $1 \%, 2 \%$ and $3 \%$, According the Eq. 10 can obtained the relationship between the $e$ and $\xi$, as it shown in Fig.4. It can concluded that, the increase of error coefficient with increase of the damping coefficient, however, the error has a same values at the point of the values of $\xi$ in 1 . This means, in the system of model, there has a minimum error in this point.

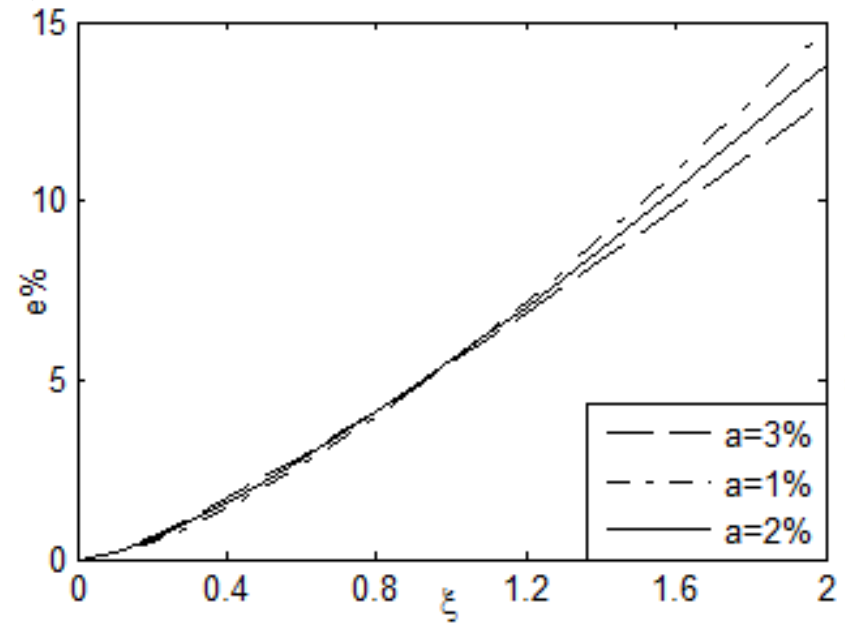

Fig. 4 Relationship between the and

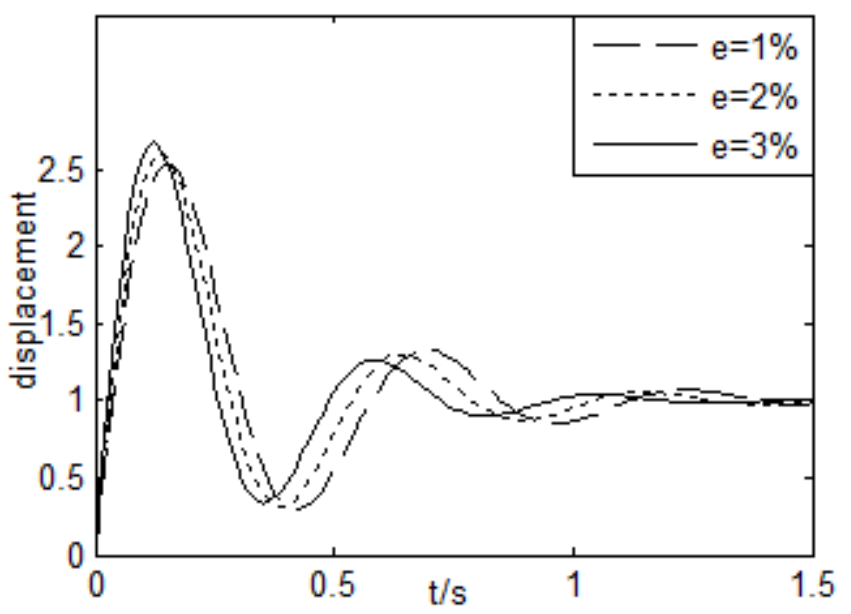

Fig. 5 Simulation of the model

Therefor, take the model into matlab for simulation with the assumption, that the damping ratio a values at 1 . Although the natural frequencies has no effect on error, the time-domain response of the force measuring system is effected by natural frequencies can be seen from Eq.9, as a result, assuming the natural frequencies is 10 . And for conform with the actual situation, we also assuming the error with the percentage at $1 \%, 2 \%$ and $3 \%$ is exists, the results of simulation is shown in Fig.6

According to the Fig. 6, the model has differents peak amplitude caused by error, and the time about peak amplitude is also effect by error. And contrast with the actual testing, which discusses in literature [9], the model is well conform with the actual situation.

\section{Conclusion}

This paper research the dynamic characteristics of seat headrest during impact by analysed the model of the impact testing. A method of three component-force detection is established in the paper and the characteristics of the forct measuring system has been analyzed; the analysis of the model error 
is researched much further. At the same time, the relationship between the damping ratio and error is been obtained. Finally, the impact model was simulated with MATLAB by computer, it is obvious that the curves are similar to the real response curves very much.

\section{Acknowledgements}

This work was financially supported by the Science and Technology Development Plan of Jilin Province ( 20130206028GX)

\section{References}

[1] GB11550-2009. Strength Requirement and Test of Automobile Seats Head Restraints. The Chinese national standard

[2] M. Monaghan.Robotic car seat testing assures durability. Automotive engineering international, Vol. 115(2):64-70.

[3] JiXiang Wang. Design of Dynamic Loading Test-bed of Head Restrains of Automobile Seat.

[4] J. M. Hurite, T. J. Kralik,M. Watanabe.Multi-axis seat durability test machine: U.S Patent 5641917 (1997)

[5] L. W. Schneider, L. L. Ricci.Survey of driver seating discomfort and related factors.1989.

[6] Guilin Zhang, Tao Jiang. Review on Tseting for Vehicle seat Static Strength. Journal of Wuhan University of Technology (Transportation Science \& Engineering)

[7] Su Chengzhi, Meng Fanyi,Cao Guohua. A Simulation Study on Dynamic Characteristics of Automotive Airbag Detection Device. Automotive Engineering 2011(03):996-999

[8] Jiang Tao, Zhang GguiLin,Li HuiMing, et al. Energy Absorption Device with Pendulum of Automobile Seats Head Restraints[J]. Applied Mechanics \& Materials, 2014, 631-632:774-778.

[9] LinYi, Yao Wei ming,Sun Dandan, Study on Automobile Seat Safety Perf ormance During Impact. Transactions of Beijing Institute of Technology,Vol.25(1) 